\title{
Superdiffusion revisited in view of collisionless reconnection
}

\author{
R. A. Treumann ${ }^{1,2,{ }^{*}}$ and W. Baumjohann ${ }^{3}$ \\ ${ }^{1}$ Department of Geophysics and Environmental Sciences, Munich University, Munich, Germany \\ ${ }^{2}$ Department of Physics and Astronomy, Dartmouth College, Hanover NH 03755, USA \\ ${ }^{3}$ Space Research Institute, Austrian Academy of Sciences, Graz, Austria \\ * currently at: International Space Science Institute, Bern, Switzerland
}

Correspondence to: R. A. Treumann (rudolf.treumann@geophysik.uni-muenchen.de)

Received: 12 January 2014 - Revised: 4 April 2014 - Accepted: 9 May 2014 - Published: 13 June 2014

\begin{abstract}
The concept of diffusion in collisionless space plasmas like those near the magnetopause and in the geomagnetic tail during reconnection is reexamined making use of the division of particle orbits into waiting orbits and breakouts into ballistic motion lying at the bottom, for instance, of Lévy flights. The rms average displacement in this case increases with time, describing superdiffusion, though faster than classical, is still a weak process, being however strong enough to support fast reconnection. Referring to two kinds of numerical particle-in-cell simulations we determine the anomalous diffusion coefficient, the anomalous collision frequency on which the diffusion process is based, and construct a relation between the diffusion coefficients and the resistive scale. The anomalous collision frequency from electron pseudo-viscosity in reconnection turns out to be of the order of the lower-hybrid frequency with the latter providing a lower limit, thus making similar assumptions physically meaningful. Tentative though not completely justified use of the $\kappa$ distribution yields $\kappa \approx 6$ in the reconnection diffusion region and, for the anomalous diffusion coefficient, the order of several times Bohm diffusivity.
\end{abstract}

Keywords. Space plasma physics (magnetic reconnection)

\section{Introduction}

Anomalous diffusion is the summary heading of all processes where the ensemble averaged mean-square displacement $\left\langle x^{2}\right\rangle \propto t^{\gamma}$ deviates from linear time dependence $\gamma=$ 1 with the classical (Einstein) diffusion coefficient $D_{\mathrm{cl}}=$ $2 T v_{\mathrm{c}} / m$, with $T$ the temperature, and $v_{\mathrm{c}}$ the classical binary collision frequency. For $\gamma>1$ one speaks of superdiffusion, which is of particular importance in the collisionless space plasma where classical diffusion is practically inhibited in all physically interesting processes (the less interesting case in space physics, $\gamma<1$, refers to subdiffusion, cf., e.g., Sokolov et al., 2002). One of those processes is reconnection, the dominant mechanism for plasma and magnetic field transport across magnetic boundaries represented by thin current sheets/layers.

Reconnection has the enormous advantage over global diffusion of being localised, with the main physics of magnetic merging and plasma mixing taking place in an extraordinarily small spatial region with the linear size shorter than the electron inertial length $<\lambda_{\mathrm{e}}=c / \omega_{\mathrm{e}}$. On this note, based on available numerical simulations, we demonstrate by estimating the anomalous collision frequency $v_{\mathrm{a}}$ that magnetic merging during reconnection can well be understood as a localised anomalous diffusion process. This result satisfactorily unifies the two originally different views on plasma transport across an apparently impermeable boundary like the magnetopause.

Anomalous diffusion is also of interest in cosmic ray physics, where it is frequently described as quasilinear diffusion resulting from wave-particle interactions, formulated in the Fokker-Planck phase space-diffusion formalism. Unfortunately, most of the observed diffusive particle spectra (cf., e.g., Christon et al., 1989, 1991, for the most elaborate observations in near-Earth space) barely exhibit the shapes resulting from quasilinear diffusion. They turn out to be power law distributions both in energy and momentum space, most frequently being described best by so-called $\kappa$ distributions

$p(\kappa \mid \mathbf{x})=A_{\kappa}\left(1+\frac{\mathbf{x}^{2}}{\kappa \ell^{2}}\right)^{-(\kappa+1+d / 2)}$ 
with normalisation factor $A_{\kappa}, d$ dimensionality, and $\ell$ correlation length (cf., e.g., Livadiotis and McComas, 2010, 2011, 2013, for an almost complete compilation of the properties of $\kappa$ distributions) with high-energy/high-momentum slopes to which the parameters $\kappa$ are related. Estimated $\kappa$ values from the magnetospheric observations range in the interval $5<\kappa<10$ (Christon et al., 1991). Such distributions were introduced by Vasyliunas (1968), following a suggestion by S. Olbert, as best fits. ${ }^{1}$ In the time-asymptotic limit, $\kappa$ distributions were explicitly derived by Hasegawa et al. (1985) and Yoon et al. (2012). Their $q$ equivalent relation to superdiffusion has also been suggested (Tsallis et al., 1995; Prato and Tsallis, 1999; Bologna et al., 2000; Gell-Mann and Tsallis, 2004, and references therein).

For the present purposes we make no direct use of these distributions as they, apparently, play no role in reconnection. Rather, as we demonstrate, anomalous diffusion in reconnection results from processes leading to waiting statistics and causing gyro-viscosity.

\section{Diffusion process}

Collisionless dissipation and related diffusion is mediated in a wider sense by collisionless turbulence (cf., e.g., Allegrini et al., 1996). Here binary collision times $\tau_{\mathrm{c}} \gg \tau_{\mathrm{a}}$ by far exceed anomalous interaction times. Any real non-collisional diffusion proceeds at times much shorter than classical (in comparison infinite) diffusion times, with absolute values of anomalous diffusion coefficients being small.

The superdiffusion process can be considered as a sequence of "waiting times" when the particle is in a quasistationary trapped state followed by "breakouts" into ballistic motion until the next trapping and waiting period starts (Shlesinger et al., 1987; Klafter et al., 1990). Such particle motions are typical, for instance, for Lévy flights (cf., e.g., Shlesinger et al., 1993).

Working in $d$ dimensions, the probability of a particle occupying a particular volume element during a process, assumed to be caused by some unspecified (nonlinear) interaction between particles and plasma waves, is most conveniently formulated in wave number space $\mathbf{k}$ with probability spectrum

\footnotetext{
${ }^{1}$ Theoretical attempts to justify solar wind $\kappa$ distributions followed, invoking wave-particle interactions with the inclusion of residual binary collisions (Scudder and Olbert, 1979). Statistical mechanical arguments were based on non-extensive statistical mechanics (Tsallis, 1988; Gell-Mann and Tsallis, 2004). From kinetic theory they were identified as collisionless turbulent quasi-stationary states far from thermal equilibrium resulting from anomalous wave-particle interactions (Treumann, 1999a, b). There the role of the temperature $T$ as a thermodynamic derivative was clarified (see also Livadiotis and McComas, 2010). The relation between the non-extensive $q$ and the $\kappa$ parameters was first given in Treumann (1997).
}

$p(\mathbf{k}) \propto \exp \left(-a k^{\alpha}\right)$,

where $a$ is some positive constant, and $0<\alpha \in \mathbb{R}$ a real number. $\alpha \geq 2$ reproduces the classical Gaussian probability spectra (Tsallis et al., 1995). Non-Gaussian spectra have flatter tails implying $\alpha<2$, indicating superdiffusion. The connection of the above probability spectrum to real-space distributions, in particular to $\kappa$ distributions, is non-trivial.

The diffusion process can be envisaged as consisting of a sequence of $n$ steps (cf., e.g., Treumann, 1997) bridging the time from $t=0$ to $t=t_{n}$ with the particle jumping from first waiting to the $n$th waiting position; the expectation value of the latter becomes

$$
\left\langle\mathbf{x}^{2}(n)\right\rangle=\int \mathbf{x}^{2} p(n \mid \mathbf{x}) d^{d} x, \quad p(n)=\prod_{1}^{n} p(i) .
$$

The $n$th expectation value is proportional to the random mean square of the displacement $\mathbf{x}^{2}$ and a power of the elapsed time sequence. Working in Fourier (or momentum) space $\mathbf{k}$, multiplication of the probabilities yields

$p(n \mid \mathbf{k})=p^{n}(\mathbf{k}) \propto \exp \left(-a n k^{\alpha}\right) \sim p\left(\mathbf{k}^{\prime}\right)$

with $p\left(\mathbf{k}^{\prime}\right)$ the probability of the $n$th time step. Hence $\mathbf{k}^{\prime}=$ $\mathbf{k} n^{1 / \alpha}$. Any real-space coordinate therefore scales as $x \rightarrow$ $x n^{-1 / \alpha}$. For the real-space probability this implies that

$p(n \mid \mathbf{x}) d^{d} x \longrightarrow p\left(\mathbf{x} / n^{1 / \alpha}\right) d^{d} x / n^{d / \alpha}$

yielding from Eq. (3) for the $n$th displacement expectation value

$\left\langle\mathbf{x}^{2}(n)\right\rangle=n^{2 / \alpha}\left\langle\mathbf{x}^{2}\right\rangle$

with $\alpha<2$ not precisely known but to be determined below from numerical simulations. The mean-square displacement should be obtained from the second moment of the underlying real-space distribution function, for instance the $\kappa$ distribution, yielding

$\left\langle\mathbf{x}^{2}\right\rangle=\frac{1}{2} d \kappa(\kappa+1) \ell^{2}$,

an expression we will make tentative (not fully justified and for the present purposes marginal) use of only at the very end in application to reconnection.

\section{Diffusion coefficient}

In using probability steps $n$, time has been discretised into pieces of free flight, waiting and some kind of interaction. On average the interaction is covered by a fictitious anomalous collision frequency $v_{\mathrm{a}}$. Ordinary binary collision frequencies $v_{\mathrm{c}}$ are very small, suggesting a scaling $v_{\mathrm{a}} \gg v_{\mathrm{c}}$ with the 
anomalous timescale $v_{\mathrm{a}}^{-1}=\tau_{\mathrm{a}} \ll \tau_{\mathrm{c}}=v_{\mathrm{c}}^{-1}$ much less than the collision timescale $\tau_{\mathrm{c}}$. The diffusion process takes place in a time $t<\tau_{\mathrm{c}}$. Replacing the time steps $n \rightarrow v_{\mathrm{a}} t$ the mean square $n$th displacement becomes

$$
\left\langle\mathbf{x}^{2}(t)\right\rangle=\left\langle\mathbf{x}^{2}\right\rangle\left(v_{\mathrm{a}} t\right)^{2 / \alpha} .
$$

With $\gamma=2 / \alpha$ it defines the anomalous diffusion coefficient $D_{\mathrm{a}}$ when multiplying by $\tau_{\mathrm{a}}^{-1}=v_{\mathrm{a}}$

$D_{\mathrm{a}}(d, t)=\left\langle\mathbf{x}^{2}\right\rangle\left(v_{\mathrm{a}} t\right)^{2 / \alpha} v_{\mathrm{a}} \equiv D_{\mathrm{ca}}\left(v_{\mathrm{a}} t\right)^{2 / \alpha}$

as a function of time $t v_{\mathrm{a}}$. Since $v_{\mathrm{a}} \gg v_{\mathrm{c}}$, it is much less than the classical diffusion coefficient which in this case would correspond to free flight. Under anomalous collisions the free flight is abruptly interrupted and reduced to non-stochastic diffusion by the finite anomalous collision frequency $v_{\mathrm{a}}$.

\section{Evolution}

Estimates of diffusion coefficients respectively $\gamma$ based on observations in space plasma are not only rare but unreliable. They suffer from the practical impossibility of any sufficiently precise determination of particle displacements as a function of time and the subsequent transition to the asymptotic state. In addition they are mostly based on quasilinear theories of particular instabilities (Sagdeev, 1966, 1979; Liewer and Krall, 1973; Huba et al., 1977, 1981; Davidson, 1978; LaBelle and Treumann, 1988; Treumann et al., 1991; Yoon et al., 2002; Matthaeus et al., 2003; Daughton et al., 2004; Ricci et al., 2005; Roytershteyn et al., 2012; Izutsu et al., 2012) which do not properly account for any nonlinear interactions.

We therefore refer to high-resolution particle-in-cell simulations (Scholer et al., 2000) performed in order to determine the cross-magnetic field diffusion of ions near quasiperpendicular shocks. The results are compiled in Fig. 1.

The right-hand side of the figure shows one macro-particle orbit arbitrarily selected out of the large number of particles used in the simulation to determine their instantaneous displacements from the origins of their trajectories in the simulation as a function of simulation time measured in units of their identical (energy-independent) gyration frequency $\omega_{\mathrm{ci}}=e B / m_{\mathrm{i}}$ in the total magnetic field, which is the sum of the ambient and the self-consistently generated turbulent wave magnetic field. The particle shifts its position perpendicular to the magnetic field from its start point to the end point in the simulation. It is found in a slowly changing waiting position, performs jumps to new waiting positions, and ends up during a final jump. Such an orbit is neither adiabatic nor stochastic.

The left part of the figure shows the average displacement, ensemble averaged over the entire particle population, as a function of simulation time. After performing an initial oscillation the average displacements settle into an approximately smooth continuously increasing curve of constant slope $\left\langle(\Delta x)^{2}\right\rangle \propto t^{1.17}$

The slope of the final evolution of the average displacement is close but by no means identical to classical diffusion, which is shown by the slope of the two straight lines in the figure. Though the deviation in the slope is small, it is nevertheless substantial and statistically significant, indicating a superdiffusive process which deviates from classical diffusion. (We should note that, because of the large number of $\sim 6.3 \times 10^{6}$ macro-particles used in the simulation of which 525000 had high energies and contributed most to the mean displacement as well as for the high time resolution, the statistical error of the measurement is smaller than the width of the line in this figure!)

Adopting the probability spectrum-based theory the experimentally determined slope of $2 / \alpha \approx 1.17$ of the average displacement in Fig. 1 tells that in these simulations one had

$\alpha \approx 1.71 \quad$ (experimental)

a value substantially far away from the Gaussian limit spectral slope $\alpha=2$ and being less than it, thus indicating quite strong superdiffusion.

\section{Transition to collisional state}

Anomalous diffusion proceeds on a faster than classical timescale with time-dependent diffusion coefficient which justifies the term superdiffusion. In spite of this, the coefficient $D_{\mathrm{ca}}=\left\langle\mathbf{x}^{2}\right\rangle v_{\mathrm{a}}$ in front of the time factor determining the absolute magnitude of the diffusion is generally small. It does not compensate for the absolute smallness of the diffusion coefficient. When, after a long time has elapsed of the order of the classical collision time $\tau_{\mathrm{c}}$, classical diffusion takes over scattering some particles to larger, some others back to smaller displacements and putting the collisionless process temporarily out of work. The average displacement of the violently scattered particles whose displacement line has been smeared out suddenly over a large spatial domain may now follow the classical linear temporary increase.

One single elapsed binary collision time may not suffice to stop the nonlinear collisionless interaction process. The widely scattered particle population may still have sufficient freedom to organise again into a softened collisionless diffusion which lasts until the next binary collision time has passed. During this second collisionless period the slope should be flatter than the initial collisionless slope, and after statistically sufficiently many periods of elapsed classical collision times no collisionless mechanisms revive anymore. Diffusion has by then become completely classical. These sequences are schematically shown in Fig. 2. 

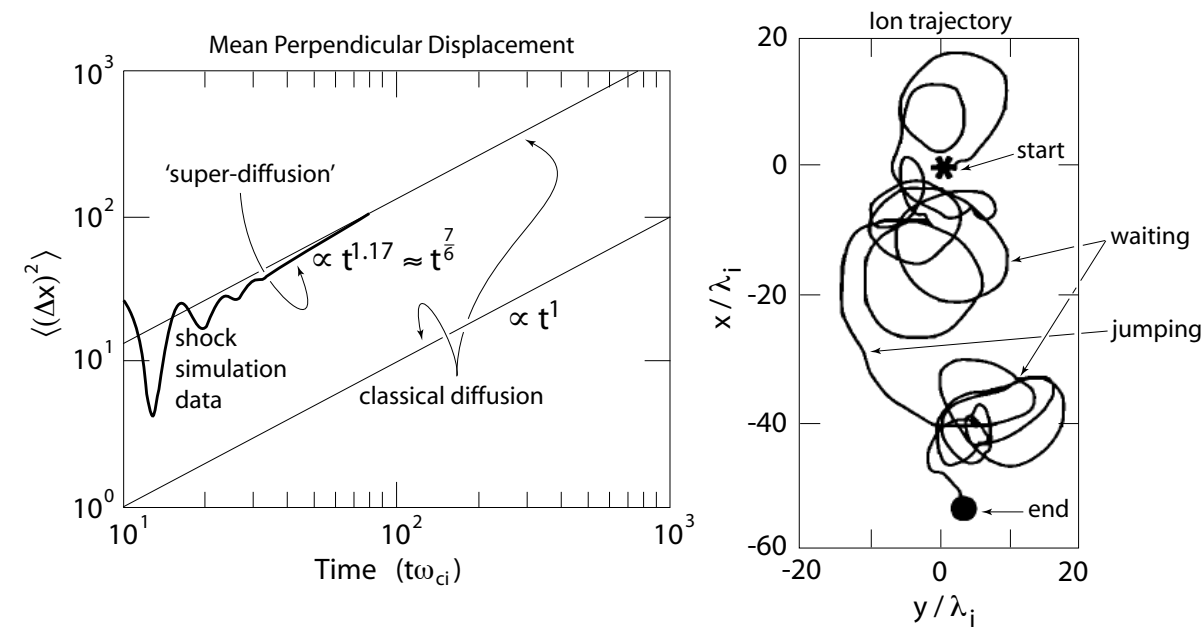

Figure 1. Two-dimensional numerical simulation results of the mean downstream perpendicular displacement of ions near a quasiperpendicular supercritical shock (shock normal angle $\theta=87^{\circ}$ ), Alfvénic Mach number $M_{\mathrm{A}}=4$ as a function of simulation time (simulation data taken from Scholer et al., 2000, courtesy American Geophysical Union). Distances are measured in ion inertial lengths $\lambda_{\mathrm{i}}=c / \omega_{\mathrm{i}}$ with $\omega_{\mathrm{i}}$ ion plasma frequency. (Left) The particle displacement performs an initial damped oscillation before settling into a continuous diffusive increase at time about $\omega_{\mathrm{ci}} t \sim 40$ (in units of the ion gyro frequency $\omega_{\mathrm{ci}}$ ). The further time evolution deviates apparently only slightly from the classical (linear) increase of the mean displacement, following a $\left\langle(\Delta x)^{2}\right\rangle \propto\left(\omega_{\mathrm{ci}} t\right)^{1.17}$ power law. (Note that simulation-time limitations did not allow monitoring of the long-time evolution of the ensemble-averaged square displacement, thus inhibiting the determination of the final state of the diffusion process.) (Right) Late time trajectory of an arbitrary ion of the sample used. The orbit is projected into the plane perpendicular to the mean magnetic field which consists of a superposition of the ambient and wave magnetic fields. The ion orbit is neither an undisturbed gyro-oscillation nor a smooth stochastic trajectory. It consists of waiting (trapped gyrating) parts and parts when the ion suddenly jumps ahead a long distance cause by some brief but intense interaction between the particle and wave spectrum. This break out of gyration is typical of rare extreme events like those in Lévy flights referred to in the present paper.

\section{Discussion}

Waiting statistics offers an approach to anomalous diffusion in various regions of space plasmas where classical (and neo-classical) diffusion processes are inappropriate, violently failing to explain the transport of plasma and magnetic fields. Application to numerical simulations near collisionless shocks determined the value of $\alpha \approx 1.71$, which turns out to be close to but sufficiently far below its classical (Gaussian) limit $\alpha=2$ for identifying superdiffusion. Superdiffusion coefficients obtained are small but increase with time.

The present theory is based on constant $\alpha$ for the entire diffusion process. This might be unrealistic. Real powers $\alpha\left[W_{\mathrm{w}}(t)\right]$ will turn out functionals of the time-dependent turbulent wave levels $W_{\mathrm{w}}(t)$ which are generated selfconsistently in the underlying turbulent collisionless waveparticle interaction (for a derivation of the phase-space distribution in particular wave-particle interactions cf., e.g., Hasegawa et al., 1985; Yoon et al., 2012, yielding timeasymptotic values of the phase-space power-law index $\kappa$ depending on wave power $W_{\mathrm{w}}$ ).

It may be expected that, with increasing wave level $W_{\mathrm{w}}(t)$, a new collisionless equilibrium will be reached where the diffusion process, in finite time $t \sim \tau_{\mathrm{f}}$, approaches another new and approximately constant diffusivity $\lim _{t \rightarrow \tau_{\mathrm{f}}} D_{\mathrm{a}}(t) \longrightarrow D_{\mathrm{a}}^{\mathrm{fin}}\left(t \gtrsim \tau_{\mathrm{f}}\right)<D_{\mathrm{c}}$

for $v_{\mathrm{a}}^{-1}(t=0) \lesssim \tau_{\mathrm{f}} \ll v_{\mathrm{c}}^{-1}$, with $W_{\mathrm{w}}\left(t \gtrsim \tau_{\mathrm{f}}\right), \alpha\left[W_{\mathrm{w}}\left(t \gtrsim \tau_{\mathrm{f}}\right)\right]$ both either constant or oscillating around their time-averaged mean values $\left\langle W_{\mathrm{w}}\left(t \gtrsim \tau_{\mathrm{f}}\right)\right\rangle,\left\langle\alpha\left(t \gtrsim \tau_{\mathrm{f}}\right)\right\rangle$, and the final average diffusion coefficient $\left\langle D_{\mathrm{a}}^{\text {fin }}\left(\tau_{\mathrm{f}} \lesssim t \lesssim v_{\mathrm{c}}^{-1}\right)\right\rangle$ remaining constant. Under such circumstances the diffusion coefficient in Fig. 2 never approaches the classical limit but settles instead on its much lower anomalous collisionless level $\left\langle D_{\mathrm{a}}^{\mathrm{fin}}\right\rangle$. The related processes lie outside the present investigation. We may, however, estimate a lower bound on the average final diffusion coefficient $\left\langle D_{\mathrm{a}}^{\mathrm{fin}}\right\rangle$ assuming $\tau_{\mathrm{f}} \approx v_{\mathrm{a}}^{-1}$, which yields

$D_{\text {ca }} \lesssim\left\langle D_{\text {a }}^{\text {fin }}\right\rangle$.

In the following we list a few practical consequences of our theory which focus on one of the most interesting problems in collisionless plasma physics, the mechanism of collisionless reconnection of magnetic fields.

\subsection{Resistive scale and relation to reconnection}

We may use these arguments to infer briefly about the resistive scale $L_{v}$, a quantity frequently referred to in discussions 


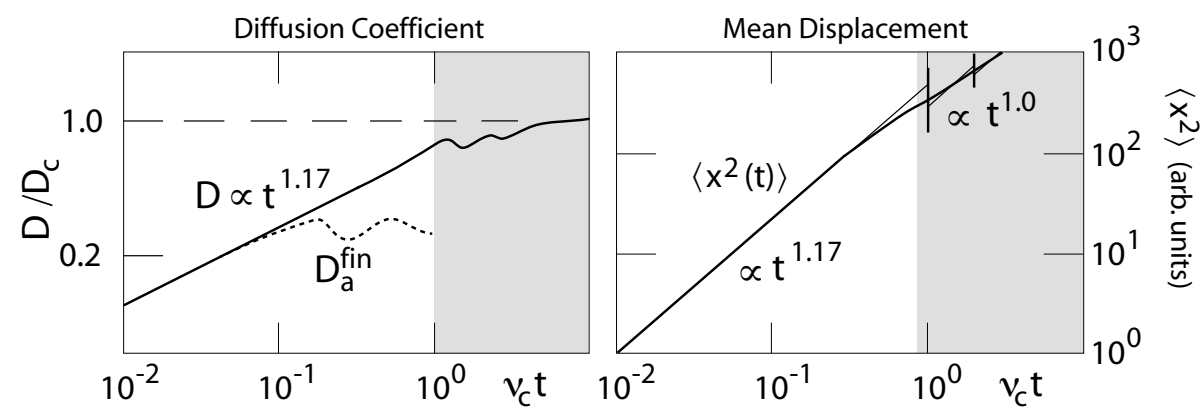

Figure 2. Schematic hypothetical evolution of the diffusion coefficient for the case simulated in Fig. 1 until the collisional classical diffusion state would have been reached. Time is measured here in classical collision times $v_{\mathrm{c}}^{-1}$. (Left) The anomalous increase of the diffusion coefficient with time. The growth of the diffusion coefficient gradually comes to rest after the classical collision time has elapsed. Dotted: A time-dependent nonlinear stationary state never approaching classical diffusion. (Right) Time evolution of the average particle displacement increasing as shown in Fig. 1. When approaching the classical collision time, scattering of particles to both larger and smaller displacements widen the displacement range, leading to a reduced increase until the second collision time. Similarly after the second, third, and the following collision times. Finally, the increase in the displacement settles into linear in time, implying classical or stationary diffusion.

of diffusion in the presence of current flow. It plays a role in the diffusive evolution of the magnetic field which from the induction equation is given in its simplest form

$$
\frac{\partial \mathbf{B}}{\partial t}=\nabla \times \mathbf{V} \times \mathbf{B}+D_{\mathrm{m}} \nabla^{2} \mathbf{B}, \quad D_{\mathrm{m}}=\frac{\eta}{\mu_{0}}=\lambda_{\mathrm{e}}^{2} \nu .
$$

The resistive scale is defined as $L_{v}^{2} \sim D_{\mathrm{m}} t=\lambda_{\mathrm{e}}^{2} \nu t$ being determined through resistivity $\eta=v / \epsilon_{0} \omega_{\mathrm{e}}^{2}$ and electron inertial length $\lambda_{\mathrm{e}}=c / \omega_{\mathrm{e}}$, with plasma frequency $\omega_{\mathrm{e}}$. It tells, at what scale resistive diffusion starts affecting the plasma dynamics.

It is interesting to know how the resistive scale evolves with time in a nonlinearly active though collisionless medium. Using the expression for the product $v_{\mathrm{a}} t$ to replace $v t$ gives

$$
\frac{L_{v_{\mathrm{a}}}}{\lambda_{\mathrm{e}}} \sim\left\{\frac{D_{\mathrm{a}}(t)}{D_{\mathrm{ca}}}\right\}^{1 / 2} \sim\left(v_{\mathrm{a}} t\right)^{1 / \alpha}
$$

for the resistive scale in units of $\lambda_{\mathrm{e}}$, expressed through the (time-dependent) diffusion coefficient $D_{\mathrm{a}}$. This indicates that the resistive scale increases with time from a value $L_{v_{\mathrm{a}}}<$ $\lambda_{\mathrm{e}}$ until $D_{\mathrm{a}} \sim\left\langle D_{\mathrm{a}}^{\mathrm{fin}}\right\rangle$ when $L_{v_{\mathrm{a}}}^{\mathrm{fin}} \sim \lambda_{\mathrm{e}}$ approaches the inertial scale.

Small (anomalous) resistive scales imply fast magnetic diffusion as observed in collisionless systems like in reconnection. Since in collisionless plasma there is no resistive diffusion, one concludes that any process causing diffusion will readily reduce the resistive scale to values below the electron inertial scale, causing comparably fast dissipation of magnetic fields and favouring reconnection.

The remaining problem consists in finding an appropriate expression for the equivalent anomalous "collision frequency" $v_{\mathrm{a}}$ under collisionless conditions. Observations (LaBelle and Treumann, 1988; Treumann et al., 1990; Bale et al., 2002) do not indicate any presence of sufficiently high wave amplitudes in collisionless reconnection required
(Sagdeev, 1966, 1979) for the quasilinear generation of anomalous resistances. Numerical particle-in-cell simulations (cf. Treumann and Baumjohann, 2013, for a recent review) confirmed instead that in all cases the main driver of fast collisionless reconnection is the electron "pseudoviscosity" implied by the presence of non-diagonal terms (Hesse and Winske, 1998; Hesse et al., 1999) in the thermally anisotropic electron pressure tensor $\mathbb{P}_{\mathrm{e}}$ measured in the stationary frame of the reconnecting current layer and accounting for any subtle finite gyro-radius effects in the dynamics of electrons in the inhomogeneous magnetic field of the electron diffusion region where electrons perform bouncing Speiser orbits.

\subsection{Gyroviscosity}

An expression for the anomalous collision frequency $v_{\mathrm{a}}$ that is equivalent to electron pseudo-viscosity is found referring to the volume viscosity $\mu_{\mathrm{V}}$ (or kinematic viscosity $\mu_{\text {kin }}=$ $\mu_{\mathrm{V}} / m N$, with $N$ the density) and the molecular collision frequency $v_{\mathrm{m}}$ (Huang, 1987)

$\mu_{\mathrm{V}}=N T / \nu_{\mathrm{m}} \quad$ or $\quad \mu_{\text {kin }}=T / m v_{\mathrm{m}}$.

Formally, this allows for the determination of $v_{\mathrm{a}}$ when identifying $\mu_{\mathrm{V}}$ with the electron volume "pseudo-viscosity" $\mu_{\mathrm{e}}$ (or kinematic pseudo-viscosity $\mu_{\mathrm{e}, \mathrm{kin}}=\mu_{\mathrm{e}} / N m_{\mathrm{e}}$ ) resulting from the non-diagonal electron pressure tensor elements, a quantity which can be determined either from observation or from numerical particle-in-cell simulations. This yields

$v_{\mathrm{a}} \approx N T_{\mathrm{e}} / \mu_{\mathrm{e}}=T_{\mathrm{e}} / m_{\mathrm{e}} \mu_{\mathrm{e}, \mathrm{kin}}$

with $N$ the plasma density and $T_{\mathrm{e}}$ the relevant electron temperature for the pressure tensor-induced equivalent anomalous collision frequency. Macmahon (1965) derived an MHD form of the full pressure tensor including finite iongyroradius contributions in the limit of very strong magnetic 
fields, barely applicable to the weak magnetic field reconnection site. A simplified version of his expressions neglecting heat fluxes was given by Stasiewicz (1987) based on the implicit assumption that in strong magnetic fields the mean free path is replaced by the ion-gyroradius. In view of reconnection, this form has been used by Hau and Sonnerup (1991) in application to rotational discontinuities (for the role of viscosities in viscous fluids, cf. Landau and Lifshitz, 1987).

In this form, rewritten for the relevant electron dynamics, one has $\mu_{\mathrm{e}} \simeq T_{\mathrm{e}} / m_{\mathrm{e}} \omega_{\mathrm{ce}}$, which identifies $v_{\mathrm{a}}=v_{\mathrm{gv}} \sim \omega_{\text {ce }}$ as an electron gyro-viscous MHD collision frequency of the order of the electron cyclotron frequency $\omega_{\mathrm{ce}}=e B / m_{\mathrm{e}}-$ indeed much larger than any Coulomb collision frequency. It suggests that gyro-viscous superdiffusion means Bohm diffusion.

\subsection{Estimates of transport quantities}

Instead, use can be made of available numerical simulations (Pritchett, 2005) which quantitatively determined the contribution of the electron-pressure tensor-induced pseudoviscosity to the dissipative generation of the parallel electric field in guide-field reconnection (cf. Treumann and Baumjohann, 2013, for a critical discussion). Pritchett (2005) obtained for the maximum non-diagonal pressure-generated field $E_{\|, P}$ in the inner part of the reconnection site (or electron exhaust region)

$E_{\|, P}=(e N)^{-1}\left|\nabla \cdot \mathbb{P}_{\mathrm{e}}\right| \lesssim 0.4 V_{\mathrm{A}} B_{0}$,

where $N, B_{0}$, and $V_{\mathrm{A}}$ are the respective density, magnetic field outside the current layer, and Alfvén velocity based on $B_{0}$. The width of the current layer was $L_{s} \sim 2 \lambda_{\mathrm{i}}=2 \sqrt{M_{s}} \lambda_{\mathrm{es}}$, with simulation mass ratio $M_{s}=m_{\mathrm{i}} / m_{\mathrm{e} s}=64$. On using in$\operatorname{dex} s$ for simulation quantities, real electron masses become $m_{\mathrm{e}}=r m_{\mathrm{e} s}$, with $r=64 / 1840$. With current $\mathbf{J}$, we may put

$E_{\|, P}=\eta_{\mathrm{as}}|\mathbf{J}| \sim \frac{\eta_{\mathrm{as}}}{\mu_{0}} \frac{B_{0}}{L_{s}}=\frac{\lambda_{\mathrm{es}} v_{\mathrm{as}} B_{0}}{2 \sqrt{M_{s}}}$

Thus, the anomalous collision frequency corresponding to the pressure-induced pseudo-viscosity in the simulation of the reconnection process was of the order of

$v_{\mathrm{a} s} \lesssim 0.8 \sqrt{M_{s}}\left(V_{\mathrm{A}} / c\right) \omega_{\mathrm{e} s}=0.8 \omega_{\mathrm{ce}, s}$

with the second form of the right-hand side resulting when accounting for the identity $\left(V_{\mathrm{A}} / c\right) \sqrt{M}=\omega_{\mathrm{ce}} / \omega_{\mathrm{e}}$. In terms of real electron masses the last expression becomes

$v_{\mathrm{a}}=v_{\mathrm{a} s} r \lesssim 0.03 \omega_{\mathrm{ce}}$.

This value is more than one order of magnitude smaller than the one of $v_{\mathrm{gv}}$ obtained above from gyro-viscous MHD theory, rewritten for electrons. Still, its value is uncertain for the unknown dependence on mass ratio of the reconnection electric field $E_{\|, P}$ in the simulations. Assuming that this dependence is moderate, the agreement is surprisingly reasonable.
For the wanted pseudo-viscosity this gives

$\mu_{\mathrm{e}, \mathrm{kin}} \approx T_{\mathrm{e}} / m_{\mathrm{e} s} v_{\mathrm{a} s}=T_{\mathrm{e}} / m_{\mathrm{e}} v_{\mathrm{a}} \gtrsim 1.25 T_{\mathrm{e}} / m_{\mathrm{e}} \omega_{\mathrm{ce}}$

with the factor $r$ in the denominator cancelling, a form similar to gyro-viscosity for both simulation and real plasma applications.

Adopting the above numerical estimate of $v_{\mathrm{a}}$, the anomalous diffusion coefficient becomes

$D_{\mathrm{a}}(t)=1.65 \times 10^{-2} D_{\mathrm{ca}}\left(\omega_{\mathrm{ce}} t\right)^{1.17}$.

It increases slowly with time measured in electron cyclotron periods.

\subsection{Digression on $\kappa$}

With the last formula we have, in principle, achieved our goal.

However, someone might want to know the explicit form of the diffusion coefficient. For this one needs to determine the coefficient $D_{\text {ca }}$, which requires knowledge of $\left\langle\mathbf{x}^{2}\right\rangle$ in the electron exhaust. Since, from the simulations, no information is available on displacements, one has to refer to model assumptions for the distribution function $p(\mathbf{x})$.

Among the limited number of such functions available one may adopt the $\kappa$ distribution Eq. (1), even though it is rather improbable that in the tiny reconnection region and for the restricted reconnection time any stationary $\kappa$ distributions will have sufficient time to evolve.

Nevertheless, in the absence of any better choice, one may tentatively evoke the relation $\alpha / 2=\kappa(\kappa+d / 2)^{-1}$ between $\alpha$ and $\kappa$, as proposed from non-extensive statistical mechanics (Tsallis et al., 1995; Prato and Tsallis, 1999; Bologna et al., 2000; Livadiotis and McComas, 2013) to hold in the superdiffusion range $\alpha<2$, and apply it as well to our particular reconnection problem.

Then, on using the measured value of $\alpha$, we have $\kappa \approx 5.9$ for $d=2$. This gives the two-dimensional $\kappa$ superdiffusion coefficient from Eqs. (22), (7), and (9), with squared correlation length $\ell^{2}=2 T_{\mathrm{e}} / m_{\mathrm{e}} v_{\mathrm{a}}$, as

$D_{\mathrm{a} \kappa}(t) \approx 11 D_{\mathrm{B}}\left(\omega_{\mathrm{ce}} t\right)^{1.17}$,

where $D_{\mathrm{B}} \approx T_{\mathrm{e}} / m_{\mathrm{e}} \omega_{\text {ce }}$ is of the order of the Bohm diffusion coefficient. This value of ten times (!) the Bohm diffusion is excessively large, implying the presence of extraordinarily strong anomalous diffusion at the reconnection site, though not in unacceptable disagreement with exceptionally fast spontaneous reconnection. For a Gaussian probability distribution one had $\left\langle x^{2}\right\rangle=\ell^{2} d / 2$ and thus $D_{\mathrm{a}}(t) \approx$ $D_{\mathrm{B}}\left(\omega_{\mathrm{ce}} t\right)^{1.17}$.

It should, however, be kept in mind that the derivation of the $\kappa$ diffusion coefficient Eq. (23) is based on the arbitrary assumption that the unknown distribution of displacements in the narrow electron exhaust would indeed be of the family of $\kappa$ distributions. While the determination of the anomalous 
collision frequency from the simulations used is very well justified, there is however no observational or any theoretical justification for this ad hoc assertion.

\subsection{Lower limit on $v_{\mathrm{a}}$ in reconnection}

The above numerical simulation-based estimates can be directly applied to observations of reconnection in the magnetotail current sheet in order to infer about the anomalous collision frequency generated in reconnection. From an applicational geophysical point of view this is most interesting. Observed magnetic fields across the tail plasma sheet vary between $1 \mathrm{nT}<B_{0}<10 \mathrm{nT}$. With these values one obtains the following range for the anomalous collision frequencies during reconnection in the plasma sheet:

$4.9 \mathrm{~Hz}<v_{\mathrm{a}}<50 \mathrm{~Hz}, \quad \omega_{\mathrm{lh}} \approx 4.1 \mathrm{~Hz}$.

These reasonably high values follow directly from analysis of the simulations, compared to the lower-hybrid frequency $\omega_{\text {lh }}$ given on the right for the lower value $B=1 \mathrm{nT}$ only. This estimated anomalous collision frequency at the magnetotail reconnection site is the result of non-stochastic processes in the electron exhaust diffusion region which generate the outof-diagonal pseudo-viscous terms in the electron pressure tensor. It is responsible for the necessary superdiffusion at the reconnection site which is required in the collisionless reconnection process.

The closeness of the lower-hybrid frequency $\omega_{\mathrm{lh}}$ to the range of anomalous collision frequencies indicates the collisionless electric coupling between electrons and ions in any reconnection process.

In addition, it provides an important lower limit

$\omega_{\mathrm{lh}} \lesssim \min _{\mathrm{rec}}\left(v_{\mathrm{a}}\right)$

on $v_{\mathrm{a}}$ in collisionless reconnection, thereby a posteriori justifying the frequently found surprising closeness (e.g. Huba et al., 1977; LaBelle and Treumann, 1988; Treumann et al., 1991; Yoon et al., 2002, and others) to the lower-hybrid frequency of the rough estimates of anomalous collision frequencies from the analysis of spacecraft observations of reconnection, which are necessary for explaining the timescale of the observed dissipation of energy.

Considered in this spirit, collisionless reconnection is understood as an equivalent anomalous local super-diffusion process in collisionless plasma. From a general physical point of view, this interpretation ultimately re-unifies the initially considered mutually excluding collisionless reconnection and diffusion theories in satisfactory concordance with fundamental electrodynamics.

Acknowledgements. This research was part of a Visiting Scientist Program at ISSI, Bern in 2006/2007. The hospitality of the librarians Andrea Fischer and Irmela Schweizer, and the technical administrator Saliba F. Saliba, is acknowledged. R. A. Treumann thanks the two anonymous referees for several helpful and clarifying comments and suggestions of related literature.

Topical Editor E. Roussos thanks P. Yoon and G. Livadiotis for their help in evaluating this paper.

\section{References}

Allegrini, P., Grigolini, P., and West, B. J.: Dynamical approach to Lévy processes, Phys. Rev. E, 54, 4760-4767, doi:10.1103/PhysRevE.54.4760, 1996.

Bale, S. D., Mozer, F. S., and Phan, T. D.: Observation of lower hybrid drift instability in the diffusion region at a reconnecting magnetopause, Geophys. Res. Lett., 29, 2180, doi:10.1029/2002GL016113, 2002.

Bologna, M., Tsallis, C., and Grigolini, P.: Anomalous diffusion associated with nonlinear fractional derivative Fokker-Plancklike equation: Exact time-dependent solutions, Phys. Rev. E, 62, 2213-2218, doi:10.1103/PhysRevE.62.2213, 2000.

Christon, S. P., Williams, D. J., Mitchell, D. G., Frank, L. A., and Huang, C. Y.: Spectral characteristics of plasma sheet ion and electron populations during undisturbed geomagnetic conditions, J. Geophys. Res., 94, 13409-13424, doi:10.1029/JA094iA10p13409, 1989.

Christon, S. P., Willams, D. J., Mitchell, D. G., Huang, C. Y., and Frank, L. A.: Spectral characteristics of plasma sheet ion and electron populations during disturbed geomagnetic conditions, J. Geophys. Res., 96, 1-22, doi:10.1029/90JA01633, 1991.

Daughton, W., Lapenta, G., and Ricci, P.: Nonlinear evolution of the lower-hybrid drift instability in a current sheet, Phys. Rev. Lett., 93, 105004, doi:10.1103/PhysRevLett.93.105004, 2004.

Davidson, R. D.: Quasi-linear stabilization of lower-hybrid-drift instability, Phys. Fluids, 21, 1373-1380, doi:10.1063/1.862379, 1978.

Gell-Mann, M. and Tsallis, C. (Eds.): Non-extensive Entropy Interdisciplinary Applications, Oxford University Press, Oxford UK, 2004.

Hasegawa, A., Mima, K., and Duong-van, M.: Plasma distribution function in a superthermal radiation field, Phys. Rev. Lett., 54, 2608-2610, doi:10.1103/PhysRevLett.54.2608, 1985.

Hau, L. N. and Sonnerup, B. U. Ö.: Self-consistent gyro-viscous fluid model of rotational discontinuities, J. Geophys. Res., 96, 15767-15778, doi:10.1029/91JA00983, 1991.

Hesse, M. and Winske, D.: Electron dissipation in collisionless magnetic reconnection, J. Geophys. Res., 103, 26479-26486, doi:10.1029/98JA01570, 1998.

Hesse, M., Schindler, K., Birn, J., and Kuznetsova, M.: The diffusion region in collisionless magnetic reconnection, Phys. Plasmas, 6, 1781-1795, doi:10.1063/1.873436, 1999.

Huang, K.: Statistical Mechanics, 2nd Edn., John Wiley \& Sons, New York, USA, Chapter 5, 1987.

Huba, J. D., Gladd, N. T., and Papadopoulos, K.: The lower-hybriddrift instability as a source of anomalous resistivity for magnetic field line reconnection, Geophys. Res. Lett., 4, 125-128, doi:10.1029/GL004i003p00125, 1977.

Huba, J. D., Gladd, N. T., and Drake, J. F.: On the role of the lower hybrid drift instability in substorm dynamics, J. Geophys. Res., 86, 5881-5884, doi:10.1029/JA086iA07p05881, 1981. 
Izutsu, T., Hasegawa, H., Nakamura, T. K. M., and Fujimoto, M.: Plasma transport induced by kinetic Alfvén turbulence, Phys. Plasmas, 19, 102305, doi:10.1063/1.4759167, 2012.

Klafter, J., Blumen, A., Zumofen, G., and Shlesinger, M. F.: Lévy walk approach to anomalous diffusion, Physica A, 168, 637-645, doi:10.1016/0378-4371(90)90416-P, 1990.

LaBelle, J. and Treumann, R. A.: Plasma waves at the dayside magnetopause, Space Sci. Rev., 47, 175-202, doi:10.1007/BF00223240, 1988.

Landau, L. D. and Lifshitz, E. M.: Fluid Mechanics, 2nd Edn., Butterworth-Heinemann, Oxford, Chapters 2 \& 5, 1987.

Liewer, P. C. and Krall, N. A.: Self-consistent approach to anomalous resistivity applied to theta pinch experiments, Phys. Fluids, 16, 1953-1963, doi:10.1063/1.1694240, 1973.

Livadiotis, G. and McComas, D. J.: Exploring transitions of space plasmas out of equilibrium, Astrophys. J., 714, 971-987, doi:10.1088/0004-637X/741/2/88, 2010.

Livadiotis, G. and McComas, D. J.: Invariant kappa distributions in space plasmas out of equilibrium, Astrophys. J., 714, 971, doi:10.1088/0004-637X/714/1/971, 2011.

Livadiotis, G. and McComas, D. J.: Understanding kappa distributions: A toolbox for space science and astrophysics, Space Sci. Rev., 175, 183-214, doi:10.1007/s11214-013-9982-9, 2013.

Macmahon, A.: Finite gyroradius corrections to the hydromagnetic equations for a Vlasov plasma, Phys. Fluids, 8, 1840-1845, doi:10.1063/1.1761116, 1965.

Matthaeus, W. H., Qin, G., Bieber, J. W., and Zank, G. P.: Nonlinear collisionless perpendicular diffusion of charged particles, Astrophys. J., 590, L53-L56, doi:10.1086/376613, 2003.

Prato, D. and Tsallis, C.: Nonextensive foundation of Lévy distributions, Phys. Rev. E, 60, 2398-2401, doi:10.1103/PhysRevE.60.2398, 1999.

Pritchett, P. L.: Onset and saturation of guide-field magnetic reconnection, Phys. Plasmas, 12, 062301, doi:10.1063/1.1914309, 2005.

Ricci, P., Brackbill, J. U., Daughton, W., and Lapenta, G.: New role of the lower-hybrid drift instability in the magnetic reconnection, Phys. Plasmas, 12, 055901, doi:10.1063/1.1885002, 2005.

Roytershteyn, V., Daughton, W., Karimabadi, H., and Mozer, F. S.: Influence of the lower-hybrid drift instability on magnetic reconnection in asymmetric configurations, Phys. Rev. Lett., 108, 185001, doi:10.1103/PhysRevLett.108.165001, 2012.

Sagdeev, R. Z.: Cooperative phenomena and shock waves in collisionless plasmas, Rev. Plasma Phys., 4, 23-91, 1966.

Sagdeev, R. Z.: The Oppenheimer lectures: Critical problems in plasma astrophysics I. Turbulence and nonlinear waves, II. Singular layers and reconnection, Rev. Mod. Phys., 51, 1-20, doi:10.1103/RevModPhys.51.1, 1979.

Scholer, M., Kucharek, H., and Giacalone, J.: Cross-field diffusion of charged particles and the problem of ion injection and acceleration at quasi-perpendicular shocks, J. Geophys. Res., 105, 18285-18293, doi:10.1029/1999JA000324, 2000.
Scudder, J. D. and Olbert, S.: A theory of local and global processes which affect solar wind electrons. I - The origin of typical $1 \mathrm{AU}$ velocity distribution functions - Steady state theory, J. Geophys. Res., 84, 2755-2772, doi:10.1029/JA084iA06p02755, 1979.

Shlesinger, M. F., West, B. J., and Klafter, J.: Lévy dynamics of enhanced diffusion - Application to turbulence, Phys. Rev. Lett., 58, 1100-1103, doi:10.1103/PhysRevLett.58.1100, 1987.

Shlesinger, M. F., Zaslavsky, G. M., and Klafter, J.: Strange kinetics, Nature, 363, 31-37, doi:10.1038/363031a0, 1993.

Sokolov, I. M., Klafter, J., and Blumen, A.: Fractional kinetics, Phys. Today, Nov. issue, 48-54, doi:10.1063/1.1535007, 2002.

Stasiewicz, K.: A gyroviscous model of the magnetotail current layer and the substorm mechanism, Phys. Fluids, 30, 1401-1409, doi:10.1063/1.866253, 1987.

Treumann, R. A.: Theory of super-diffusion for the magnetopause, Geophys. Res. Lett., 24, 1727-1730, doi:10.1029/97GL01760, 1997.

Treumann, R. A.: Kinetic theoretical foundation of Lorentzian statistical mechanics, Phys. Scr., 59, 19-26, doi:10.1238/Physica.Regular.059a00019, 1999a.

Treumann, R. A.: Generalized-Lorentzian thermodynamics, Phys. Scr., 59, 204-214, doi:10.1238/Physica.Regular.059a00204, $1999 b$.

Treumann, R. A. and Baumjohann, W.: Collisionless magnetic reconnection in space plasmas, Front Physics, 1, 00031 , doi:10.3389/fphy.2013.00031, 2013.

Treumann, R. A., Sckopke, N., Brostrom, L., and LaBelle, J.: The plasma wave signature of a magnetic hole' in the vicinity of the magnetopause, J. Geophys. Res., 95, 19099-19114, doi:10.1029/JA095iA11p19099, 1990.

Treumann, R. A., LaBelle, J., and Pottelette, R.: Plasma diffusion at the magnetopause - The case of lower hybrid drift waves, J. Geophys. Res., 96, 16009-16013, doi:10.1029/91JA01671, 1991.

Tsallis, C.: Possible generalization of Boltzmann-Gibbs statistics, J. Stat. Phys., 52, 479-487, doi:10.1007/BF01016429, 1988.

Tsallis, C., de Souza, A. M. C., and Maynard, R.: Derivation of Lévy-type anomalous superdiffusion from generalized statistical mechanics, Lect. Notes Phys., 450, 269-289, 1995.

Vasyliunas, V. M.: A survey of low-energy electrons in the evening sector of the magnetosphere with OGO 1 and OGO 3, J. Geophys. Res., 73, 2839-2884, doi:10.1029/JA073i009p02839, 1968.

Yoon, P. H., Lui, A. T. Y., and Sitnov, M. I.: Generalized lowerhybrid drift instabilities in current-sheet equilibrium, Phys. Plasmas, 9, 1526-1538, doi:10.1063/1.1466822, 2002.

Yoon, P. H., Ziebell, L. F., Gaelzer, R., Lin, R. P., and Wang, L.: Langmuir turbulence and suprathermal electrons, Space Sci. Rev., 173, 459-489, doi:10.1007/s11214-012-9867-3, 2012. 